\title{
Structured Coprime Factor Model Reduction based on LMIs *
}

\author{
$\mathrm{Li} \mathrm{Li}^{\text {a }}$, Fernando Paganini ${ }^{\mathrm{a}, 1}$ \\ ${ }^{a}$ Electrical Engineering Department, University of California, Los Angeles, Box 951594, Los Angeles, CA 90095-1594
}

\begin{abstract}
In this paper we discuss dynamic model reduction methods which preserve a certain structure in the underlying system. Specifically, we consider the situation where the reduction must be consistent with a partition of the system states. This is motivated, for instance, in situations where state variables are associated with the topology of a networked system, and the reduction should preserve this. We build on the observation that imposing block structure to generalized controllability and observability gramians automatically yields such state-partitioned model reduction. The difficulty lies in ensuring feasibility of the resulting Lyapunov inequalities, which is in general very restrictive. To overcome this, we consider coprime factor model reduction. We derive an LMI characterization of expansive and contractive coprime factorizations that preserve structure, and use this to build a more flexible method for structured model reduction. An example is given to illustrate the method.
\end{abstract}

Key words: Model reduction; Structure preserving; Coprime factorization; Linear matrix inequality(LMI); Algebraic Riccati inequality

\section{Introduction}

Methods for obtaining lower-order approximations of linear state-space systems have been in place since the early 80s (Moore, 1981; Enns, 1984; Glover, 1984), and yield bounds on model reduction error from an inputoutput perspective. Superficially, such methods would appear attractive for the study of large-scale systems and networks which suffer from the curse of state dimensionality. However, a closer look reveals an important difficulty: these methods would destroy the topological structure which is inherent in large-scale systems. By topological structure we mean that these models are naturally built from interconnections of subsystem models, and therefore state variables, inputs and outputs are associated with specific subsystems or network regions: for many applications, it is desirable to maintain such identification for the reduced system states. The above-mentioned methods, in contrast, rely on global changes of state coordinates (e.g., balancing), implying the reduced state variables can only be given a global interpretation. As an example, consider the power grid,

\footnotetext{
* This paper was not presented at any IFAC meeting. Research supported by EPRI/DoD Grant WO-8333-06, and NSF Grant ECS-9875056.

Email addresses: lleely@ee.ucla.edu ( $\mathrm{Li} \mathrm{Li})$, paganini@ee.ucla.edu (Fernando Paganini).

1 Corresponding author, Tel: (310) 206-1426, Fax: (310) 2064685 .
}

a network obtained by interconnection of various regional subnetworks made of power plants, substations and other devices. Typical state variables for a certain subsystem could be rotor angles and speed in generators, or electrical quantities in local lines or other equipment. Inputs and outputs used for control (e.g., voltage stabilizers) are also local to each region. A reduced model of the entire network can summarize many of the states into fewer variables, and thus simplify design and simulation, but should still respect region boundaries, to provide engineers (which could even belong to different organizations) with reduced models with local meaning (see Tsai, Narasimhamurthi, \& Wu 1982 for more discussion of the power grid problem).

Motivated by this issue, in this paper we consider a statespace system of transfer function $G(s)$,

$$
\dot{x}=A x+B u, \quad y=C x+D u,
$$

where the state vector is broken in $N$ sub-vectors $x=$ $\left(x_{1}^{T}, \ldots, x_{N}^{T}\right)^{T}$, each associated with a different subsystem, and inputs and outputs are also partitioned accordingly. We want the reduced system's states to maintain this association. For simplicity of exposition, we will consider a partition of only two groups of states, where

$$
A=\left[\begin{array}{ll}
A_{11} & A_{12} \\
A_{21} & A_{22}
\end{array}\right], B=\left[\begin{array}{cc}
B_{1} & 0 \\
0 & B_{2}
\end{array}\right], C=\left[\begin{array}{cc}
C_{1} & 0 \\
0 & C_{2}
\end{array}\right], D=\left[\begin{array}{cc}
D_{1} & 0 \\
0 & D_{2}
\end{array}\right]
$$

with conformal dimension. This amounts to having two subsystems with separate inputs and outputs, but interacting through the dynamics of their respective states 
$x_{1}, x_{2}$. We want the operations performed to go from $G$ to the reduced model $G_{r}$ to be such that the final model has a state variable $x_{r}=\left(x_{r 1}^{T}, x_{r 2}^{T}\right)^{T}$, where $x_{r 1}$ is only a function of $x_{1}, x_{r 2}$ is a function of $x_{2}$, and the inputs and outputs remain decoupled.

Linear Matrix Inequality (LMI) approaches to the model reduction problem (see e.g, Dullerud \& Paganini, 2000) open the door for obtaining such property, by imposing block structure on generalized system gramians. Indeed, this idea has already been pursued in Zhou, D'Souza, \& Cloutier (1995) for a plant-controller interconnection, and in Beck, Doyle, \& Glover (1996) for multi-dimensional systems, where the state partitions correspond to different frequency variables, or uncertainty variables. We will consider the structure

$\mathcal{G}_{s}=\left\{H \mid H=H^{*}=\operatorname{diag}\left(H_{1}, H_{2}\right)\right.$ consistent with (1) $\}$

imposed on solutions to the Lyapunov inequalities

$A X+X A^{*}+B B^{*}<0, A^{*} Y+Y A+C^{*} C<0$

for the generalized gramians $X>0$ and $Y>0$. Such a block-diagonal structure on these variables automatically forces the resulting balancing transformations to respect the subsystem boundaries, and thus the reduced order model will maintain a topological association.

The main difficulty in applying this method is that only under some very strong conditions will (2) be feasible with block diagonal $X, Y$; in particular it is not enough to have stability of $A$. For instance, lightly-damped springmass systems which are essentially the models of powergrid oscillations would not fall in this category. To avoid this problem, in this paper we seek structured model reduction methods in the coprime factor description of a system. In the absence of structure, such methods have been developed by Anderson \& Liu (1989); Liu \& Anderson (1986); McFarlane \& Glover (1990); Meyer (1990); El-Zobaidi \& Jaimoukha (1998), and can be applied even to unstable systems; they are briefly reviewed in Section 2. Particularly attractive are reductions based on normalized coprime factorizations, since robustness results on closed-loop stability are available. However normalization would also destroy our topological structure, so we use again LMI conditions to produce suitable relaxations for which the structure can be imposed. Two approaches are presented in Section 3, based respectively on expansive and contractive factorizations. We illustrate the methods by a mass-spring example presented in Section 4. Conclusions are given in Section 5. A preliminary version of this work appeared in Li \& Paganini (2002), and frequency-weighted extensions are also done by $\mathrm{Li}$ \& Paganini (2003).

\section{Background}

In what follows, we adopt the following standard notation. Let $M$ be a complex matrix, then $M^{*}$ denotes the complex conjugate transpose of $M$, and $\bar{\sigma}(M)$ the largest singular value of $M$. The state-space realization of a transfer matrix is denoted by $G(s)=\left[\frac{A \mid B}{C \mid D}\right]:=$ $C(s I-A)^{-1} B+D$. The $\mathcal{H}_{\infty}-$ norm of $G(s)$ is denoted by $\|G\|_{\infty}=\sup _{\omega} \bar{\sigma}(G(j \omega))$. The collection of all rational proper and stable transfer matrices is denoted by $\mathcal{R H}_{\infty}$.

We first review balanced truncation using generalized gramians (Dullerud \& Paganini, 2000), i.e. any $X, Y$ satisfying (2) ${ }^{2}$. A state transformation $T$ leads to the transformed gramians $\tilde{X}=\tilde{Y}=\operatorname{diag}\left[\gamma_{1}, \ldots, \gamma_{n}\right]$; the diagonal elements $\gamma_{i}$ are called the generalized Hankel singular values. A subsequent truncation of the state to order $k$ gives the following error bound.

Proposition 1 If $G=\left[\frac{A \mid B}{C \mid 0}\right]$ is minimal, with A Hurwitz and the generalized Hankel singular values $\gamma_{1} \geq$ $\ldots \geq \gamma_{k}>\gamma_{k+1} \geq \ldots \geq \gamma_{n}$, and $G_{r}=\left[\begin{array}{c|c}A_{r} & B_{r} \\ \hline C_{r} \mid 0\end{array}\right]$ is obtained by $k$-th order generalized balanced truncation, then $A_{r}$ is Hurwitz and $\left\|G-G_{r}\right\|_{\infty} \leq 2\left(\gamma_{1}^{t}+\cdots+\gamma_{q}^{t}\right)$, where $\gamma_{i}^{t}$ denote the distinct generalized Hankel singular values of $\gamma_{k+1}, \ldots, \gamma_{n}$, that is, $\gamma_{1}^{t}>\gamma_{2}^{t}>\ldots>\gamma_{q}^{t}$ and $\left\{\gamma_{k+1}, \ldots, \gamma_{n}\right\}=\left\{\gamma_{1}^{t}, \ldots, \gamma_{q}^{t}\right\}$

We now review some classical work on model reduction via normalized coprime factorizations, which can be found in McFarlane \& Glover (1990); Zhou, Doyle, \& Glover (1996). A right coprime factorization (RCF) $(M, N)$ of plant $G$ with $G=N M^{-1}$ is called normalized if $\left[\begin{array}{l}N \\ M\end{array}\right]$ is inner, i.e. if $N, M \in \mathcal{R} \mathcal{H}_{\infty}$ and $N(j \omega)^{*} N(j \omega)+$ $M(j \omega)^{*} M(j \omega)=I$ for all $\omega$. The following characterizes normalization in terms of the LQ Riccati equation.

Theorem 2 Given $G(s)=\left[\frac{A \mid B}{C \mid D}\right]$, suppose $(A, B)$ is stabilizable and $(C, A)$ has no unobservable modes on the imaginary axis. Denote $R=I+D^{*} D, S=I+D D^{*}$, then a normalized $R C F$ of $G$ is given by

$$
\left[\begin{array}{c}
N(s) \\
M(s)
\end{array}\right]=\left[\begin{array}{c|c}
A+B F & B R^{-\frac{1}{2}} \\
\hline C+D F & D R^{-\frac{1}{2}} \\
F & R^{-\frac{1}{2}}
\end{array}\right],
$$

where $F=-R^{-1}\left(B^{*} X_{s}+D^{*} C\right)$, and $X_{s}$ is the stabilizing solution of the Algebraic Riccati Equation

$$
\begin{gathered}
X_{s}\left(A-B R^{-1} D^{*} C\right)+\left(A-B R^{-1} D^{*} C\right)^{*} X_{s} \\
-X_{s} B R^{-1} B^{*} X_{s}+C^{*} S^{-1} C=0 .
\end{gathered}
$$

Since coprime factors are stable, standard reduction methods such as balanced truncation apply to them. The advantage of a normalized factorization is that, given a stabilizing controller for the plant $G$, it allows one to quantify the robust stability margin, i.e. the

\footnotetext{
${ }^{2}$ If they exist, these would be non-unique, a fact that can be exploited for further optimization, see below.
} 
maximum uncertainty in the coprime factors for which the controller is stabilizing.

Lemma 3 (McFarlane $\&$ Glover, 1990; Dullerud \& Paganini, 2000) If controller $K$ stabilizes $G=N M^{-1}$, where $(M, N)$ is normalized $R C F$ of $G$, then $K$ stabilizes $G_{\triangle}=\left(N+\triangle_{N}\right)\left(M+\triangle_{M}\right)^{-1}$ for all $\left[\begin{array}{c}\triangle_{N} \\ \triangle_{M}\end{array}\right] \in \mathcal{R} \mathcal{H}_{\infty}$ and $\left\|\left[\begin{array}{c}\triangle_{N} \\ \triangle_{M}\end{array}\right]\right\|_{\infty} \leq \epsilon$ iff $\left\|\left[\begin{array}{c}G \\ I\end{array}\right](I-K G)^{-1}\left[\begin{array}{ll}K & I\end{array}\right]\right\|_{\infty}^{-1}>\epsilon$.

The accuracy of approximation in the normalized coprime factor model reduction is usually chosen in regard to a certain closed loop stability consideration, invoking Lemma 3. For instance, one could use this procedure to design reduced-order stabilizing controllers for a high order system in one of two ways (see McFarlane \& Glover, 1990): firstly, reduce the plant, design the corresponding low-order controller to minimize $\left\|\left[\begin{array}{c}G \\ I\end{array}\right](I-K G)^{-1}\left[\begin{array}{ll}K & I\end{array}\right]\right\|_{\infty}$ in Lemma 3, and check if the stability margin it gives allows one to ensure stability of the original plant. Otherwise iterate; secondly, design a full-order controller, find its stability margin, and then reduce the controller's coprime factors to an accuracy allowed by the margin. We remark as well that this robustness property is related to the fact that right coprime factors generate the graph of the system (i.e. the set of input-output pairs in $L_{2}$ space), and (see e.g. Georgiou \& Smith, 1990; Vinnicombe, 1993) approximating such graphs is key to preserving stability.

\section{LMI-Based Algorithm for Structured Co- prime Factor Model Reduction}

We want to impose structure in an approximation method such as normalized coprime factor reduction; as explained in the introduction, we can preserve the partition of system states by imposing the structure $\mathcal{G}_{s}$ on the generalized controllability and observability gramians of the plant RCF. However we also want to preserve decoupled inputs and outputs; looking closely at normalized coprime factor reduction, we see that unless the feedback $F$ for the normalized RCF is decentralized (block-diagonal) the input-output structure will not remain decoupled between subsystems. To impose this decentralization, we need a generalization of normalized RCFs which will allow more flexibility, but at the same time approximate the normalization requirement. The main idea of this paper is to work with LMI relaxations of the LQ Riccati equation used for finding normalized RCFs. One type of relaxation yields expansive factorizations (defined below), which is attractive since one can generalize the robustness results, however stability of the factorization is not automatically imposed, and thus the overall problem becomes non-convex. The opposite relaxation, contractive factorization, has more attractive computational properties, but then a heuristic is required to induce approximate normalization. The two approaches are now discussed, and we begin by defining an expansive RCF of an LTI system.

Definition 4 A RCF of $G=N M^{-1}$ with $N, M \in \mathcal{R} \mathcal{H}_{\infty}$ is expansive if $N(j \omega)^{*} N(j \omega)+M(j \omega)^{*} M(j \omega) \geq I, \forall \omega$.

From the above definition it is routinely shown that the sufficiency part of Lemma 3 still holds for an expansive factorization, consequently a stability margin can also be predetermined; this is the main motivation for considering expansive factorizations. For related work in a multi-dimensional setting, see Beck \& Bendottii (1997). The following theorem indicates a procedure to seek expansive factorizations. The proof is very similar to the one for contractive factorizations, covered below, and hence is omitted (it can be found in Li \& Paganini 2002).

Theorem 5 Given $G(s)=\left[\frac{A \mid B}{C \mid D}\right]$, let $R=I+D^{*} D$, $S=I+D D^{*}$, and $X_{e}$ satisfy the $L M I$

$\left[\begin{array}{cc}X_{e}\left(A-B R^{-1} D^{*} C\right)+\left(A-B R^{-1} D^{*} C\right)^{*} X_{e}+C^{*} S^{-1} C & X_{e} B \\ B^{*} X_{e} & R\end{array}\right]$

Also let $F_{e}=-R^{-1}\left(B^{*} X_{e}+D^{*} C\right)$ and

$$
U(s)=\left[\begin{array}{c}
N(s) \\
M(s)
\end{array}\right]=\left[\begin{array}{c|c}
A+B F_{e} & B R^{-\frac{1}{2}} \\
\hline C+D F_{e} & D R^{-\frac{1}{2}} \\
F_{e} & R^{-\frac{1}{2}}
\end{array}\right] .
$$

Then $G(s)=N(s) M(s)^{-1}$ and $U(j \omega)^{*} U(j \omega) \geq I$ for all $j \omega \notin \operatorname{eig}\left(A+B F_{e}\right)$.

Note that Theorem 5 does not guarantee the stability of $A+B F_{e}$, therefore $(M, N)$ is so far not yet an expansive factorization by Definition 4 . Moreover, we do not only want stability but something stronger: namely, the coprime factorization must admit structured generalized controllability and observability gramians. More precisely, the condition

$\left(A-B R^{-1}\left(B^{*} X_{e}+D^{*} C\right)\right) Y_{e}+Y_{e}\left(A-B R^{-1}\left(B^{*} X_{e}+D^{*} C\right)\right)^{*}<0$

should admit a structured solution $Y_{e} \in \mathcal{G}_{s}$.

Unfortunately, the two conditions (4-5) are not jointly convex in $X_{e}, Y_{e}$ (they are a BMI), and so we do not have a tractable method to determine conclusively its feasibility. In Li \& Paganini (2002) we used a two-step procedure to seek solutions for it: first, use a heuristic to make the solution $X_{e}$ of (4) as close to the stabilizing solution of (3) as possible, and then check for feasibility of (5) as an LMI in $Y_{e}$. The behavior of this heuristic method will be demonstrated in an example in Section 4.

We turn now to the opposite relaxation to the normalization requirement: namely, using contractive RCFs.

Definition 6 A RCF of $G=N M^{-1}$ with $N, M \in \mathcal{R} \mathcal{H}_{\infty}$ is contractive if $N(j \omega)^{*} N(j \omega)+M(j \omega)^{*} M(j \omega) \leq I, \forall \omega$. 
Lemma 7 Given $G(s)=\left[\frac{A \mid B}{C \mid D}\right], X$ satisfies $A^{*} X+$ $X A+C^{*} C<0$ and $C^{*} D+X B=0$, then $G(j \omega)^{*} G(j \omega) \leq$ $D^{*} D$ for all $j \omega \notin \operatorname{eig}(A)$.

PROOF. Let $G(s)=\left[\frac{-A^{*} \mid-C^{*}}{B^{*}} \mid D^{*}\right]$, then $G(j \omega)=$ $G(j \omega) *$ for all $j \omega \notin e i g(A)$. We obtain

$$
G(s) G(s)=\left[\begin{array}{cc|c}
-A^{*}-C^{*} C & -C^{*} D \\
0 & A & B \\
\hline B^{*} D^{*} C & D^{*} D
\end{array}\right] .
$$

Let $Q=A^{*} X+X A+C^{*} C<0$, and introduce the state transformation $T=\left[\begin{array}{cc}I & -X \\ 0 & I\end{array}\right]$ which leads to $G(s) G(s)=-B^{*}\left(s I+A^{*}\right)^{-1} Q(s I-A)^{-1} B+D^{*} D$

by using $C^{*} D+X B=0$. Let $s=j \omega$, we get

$$
\begin{aligned}
G(j \omega)^{*} G(j \omega) & =\left[(j \omega I-A)^{-1} B\right]^{*} Q\left[(j \omega I-A)^{-1} B\right]+D^{*} D \\
& \leq D^{*} D \quad \forall j \omega \notin e i g(A) .
\end{aligned}
$$

We now state the convex condition to search for contractive factorizations (similar conditions appear in Wood, Goddard, \& Glover 1996; El-Zobaidi \& Jaimoukha 1998 in the context of LPV systems).

Theorem 8 Given $G(s)=\left[\frac{A \mid B}{C \mid D}\right]$, let $R=I+D^{*} D$, $S=I+D D^{*}$, and $\bar{X}_{c}>0$ satisfy

$\left[\begin{array}{cc}\left(A-B R^{-1} D^{*} C\right) \bar{X}_{c}+\bar{X}_{c}\left(A-B R^{-1} D^{*} C\right)^{*}-B R^{-1} B^{*} \bar{X}_{c} C^{*} \\ C \bar{X}_{c} & -S\end{array}\right]$

Also let $F_{c}=-R^{-1}\left(B^{*} \bar{X}_{c}^{-1}+D^{*} C\right)$ and

$$
U(s)=\left[\begin{array}{c|c}
N(s) \\
M(s)
\end{array}\right]=\left[\begin{array}{c|c}
A+B F_{c} & B R^{-\frac{1}{2}} \\
\hline C+D F_{c} & D R^{-\frac{1}{2}} \\
F_{c} & R^{-\frac{1}{2}}
\end{array}\right]
$$

then $(N(s), M(s))$ is a contractive $R C F$ of $G(s)$.

PROOF. By Schur complement and changing variable with $X_{c}=\bar{X}_{c}^{-1}$, (6) is equivalent to

$$
\begin{gathered}
X_{c}\left(A-B R^{-1} D^{*} C\right)+\left(A-B R^{-1} D^{*} C\right)^{*} X_{c} \\
-X_{c} B R^{-1} B^{*} X_{c}+C^{*} S^{-1} C<0
\end{gathered}
$$

which leads to

$X_{c}\left(A+B F_{c}\right)+\left(A+B F_{c}\right)^{*} X_{c}+\left(\begin{array}{c}C+D F_{c} \\ F_{c}\end{array}\right)^{*}\left(\begin{array}{c}C+D F_{c} \\ F_{c}\end{array}\right)<0$

Thus $A+B F_{c}$ is Hurwitz. Notice that

$$
\left(\begin{array}{c}
C+D F_{c} \\
F_{c}
\end{array}\right)^{*}\left(\begin{array}{c}
D R^{-\frac{1}{2}} \\
R^{-\frac{1}{2}}
\end{array}\right)+X_{c} B R^{-\frac{1}{2}}=0,
$$

by Lemma $7, U(j \omega)^{*} U(j \omega) \leq I, \forall j \omega \notin e i g\left(A+B F_{c}\right)$.

Note that Theorem 8 guarantees the stability of $A+$ $B F_{c}$, therefore no other restriction is needed as in the expansive method. So if (6) is feasible with the additional restriction $\bar{X}_{c} \in \mathcal{G}_{s}$, this already produces a contractive factorization which preserves the input-output structure, and which will admit, furthermore, generalized gramians with the correct structure. Indeed, writing the Lyapunov inequalities for the contractive factorization $U(s)$ in the unknowns $P_{c}, Q_{c}$, we have:

$$
\begin{gathered}
\left(A+B F_{c}\right) P_{c}+P_{c}\left(A+B F_{c}\right)^{*}+B R^{-1} B^{*}<0 \quad(10 \mathrm{a}) \\
\left(A+B F_{c}\right)^{*} Q_{c}+Q_{c}\left(A+B F_{c}\right)+\left(\begin{array}{c}
C+D F_{c} \\
F_{c}
\end{array}\right) *\left(\begin{array}{c}
C+D F_{c} \\
F_{c}
\end{array}\right)<0
\end{gathered}
$$

The feasibility of (10b) follows directly from (9) by letting $Q_{c}=X_{c}$, implying $A+B F_{c}$ has the necessary stability to allow for structured generalized gramians, and therefore (10a) will be feasible as well. So, provided (6) is feasible, we can proceed with our structured method.

What we give up in this version are the robustness guarantees of the normalized (or expansive) method; in fact, if we want a robustness theorem in this case it should be based on a certain level of " $\gamma$-expansiveness", as in

$$
I \geq N(j \omega)^{*} N(j \omega)+M(j \omega)^{*} M(j \omega) \geq \gamma^{2} I
$$

Since such a condition is difficult to impose, we will instead use a heuristic to try to make our contractive factorization approach the normalized one. To explore this, we first review the relation between $X_{c}$ in (8) and the stabilizing solution of the control Riccati equation.

Lemma 9 Let $X_{s}$ be denoted the stabilizing solution of (3) and $X_{c}>0$ satisfy (8), then $X_{c}>X_{s}$.

PROOF. Subtract (3) from (8),

$$
\begin{gathered}
\left(X_{c}-X_{s}\right)\left(A+B F_{c}\right)+\left(A+B F_{c}\right)^{*}\left(X_{c}-X_{s}\right) \\
<-\left(X_{s} B-X_{c} B\right) R^{-1}\left(X_{s} B-X_{c} B\right)^{*} \leq 0 .
\end{gathered}
$$

Since $A+B F_{c}$ is Hurwitz from Theorem 8, $X_{c}>X_{s}$.

Based on this condition, we see that in the absence of structure, a normalized coprime factorization could be obtained by minimizing $X_{c}$ subject to (8), or equivalently minimizing $\bar{X}_{c}^{-1}$ subject to (6). Thus a possible heuristic to seek approximate normalization in the presence of structure is the semidefinite program

$$
\begin{aligned}
& \min \operatorname{trace}(Z) \text { with } \bar{X}_{c} \in \mathcal{G}_{s} \\
& \text { subject to }(6) \text { and }\left[\begin{array}{cc}
\bar{X}_{c} & I \\
I & Z
\end{array}\right]>0
\end{aligned}
$$

After $\bar{X}_{c}$ is found, its inverse $X_{c}$ is a generalized observability gramian $Q_{c}$ of the coprime factors. To obtain $P_{c}$, one possibility would be to solve (10a) in a second step by minimizing its trace to approximate the true gramian.

An alternative, in which both generalized gramians can be found in one step, is based on considering the dual, filter Riccati inequality

$$
\begin{gathered}
\left(A-B R^{-1} D^{*} C\right) Y_{c}+Y_{c}\left(A-B R^{-1} D^{*} C\right)^{*} \\
-Y_{c} C^{*} S^{-1} C Y_{c}+B R^{-1} B^{*}<0 .
\end{gathered}
$$




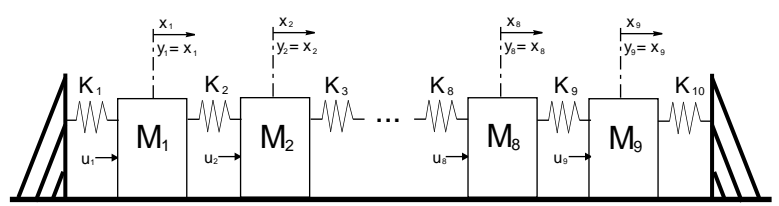

Fig. 1. 9-mass spring system.

The following lemma (El-Zobaidi \& Jaimoukha, 1998) shows the connection between the generalized gramians of the contractive factorization and the solutions of the Riccati inequalities (8), (13).

Lemma 10 Let $U(s)$ represent the contractive $R C F$ of $G(s)=\left[\frac{A \mid B}{C \mid D}\right]$, given by (7). Let $X_{c}>0$ solve (8) and $Y_{c}>0$ solve (13), then $P_{c}=Y_{c}\left(I+X_{c} Y_{c}\right)^{-1}$ and $Q_{c}=X_{c}$ are one pair of generalized controllability and observability gramians for $U(s)$.

A suitable $Y_{c} \in \mathcal{G}_{s}$ could be found separately, based on $\left[\begin{array}{cr}\bar{Y}_{c}\left(A-B R^{-1} D^{*} C\right)+\left(A-B R^{-1} D^{*} C\right)^{*} \bar{Y}_{c}-C^{*} S^{-1} C & \bar{Y}_{c} B \\ B^{*} \bar{Y}_{c} & -R\end{array}\right]<0$

with $\bar{Y}_{c}=Y_{c}^{-1}$, equivalent to (13), and then used to construct $P_{c}$. Any solution of (14) will do, but once more it is natural here to solve the following problem

$$
\begin{aligned}
& \min \operatorname{trace}(W) \text { with } \bar{Y}_{c} \in \mathcal{G}_{s} \\
& \text { subject to }(14) \text { and }\left[\begin{array}{cc}
\bar{Y}_{c} & I \\
I & W
\end{array}\right]>0
\end{aligned}
$$

since in the absence of a structure restriction (15) and (12) would lead to the actual gramians of the contractive factorization. We now summarize the overall procedure.

\section{Procedure 11 (Contractive RCF Model Reduction)}

(1) Solve the semidefinite programs (12) and (15).

(2) Write $U$ of degree $n$, with the form of (7).

(3) Let $P_{c}=\operatorname{diag}\left(P_{1}, P_{2}\right)=\left(\bar{Y}_{c}+\bar{X}_{c}^{-1}\right)^{-1}$ and $Q_{c}=$ $\operatorname{diag}\left(Q_{1}, Q_{2}\right)=\bar{X}_{c}^{-1}$ be generalized gramians of $U$, balance $\left(P_{i}, Q_{i}\right)$ by $T_{i}, i=1,2$, to get the transformation matrix $T=\operatorname{diag}\left(T_{1}, T_{2}\right)$.

(4) The new balanced system is $U_{\text {new }}=\left[\begin{array}{l|l}A_{\text {new }} & B_{\text {new }} \\ \hline C_{\text {new }} & D_{\text {new }}\end{array}\right]$ with balanced generalized gramian $\Sigma=\operatorname{diag}\left(\Sigma_{1}, \Sigma_{2}\right)$, where $\Sigma_{i}=\operatorname{diag}\left(\Sigma_{i 1}, \Sigma_{i 2}\right)$. The sub-matrices of $A_{\text {new }}, B_{\text {new }}, C_{\text {new }}$ corresponding to $\Sigma_{i 2}$ are truncated to obtain the reduced order approximation $U_{r}=\left[\begin{array}{l}N_{r} \\ M_{r}\end{array}\right]$ of degree $k<n$.

(5) Form the reduced plant $G_{r}=N_{r} M_{r}^{-1}$ of degree $k$.

\section{Example}

We consider the 9-mass physical system shown in Fig. 1. The states are the position and velocity of each mass; there is a force input and a position output at each mass, and some friction with the ground. Specifically, the dynamics of each mass $M_{i}, i=1, \ldots, 9$, satisfies

$$
\begin{aligned}
\dot{x}_{i} & =v_{i}, \\
M_{i} \dot{v}_{i} & =K_{i}\left(x_{i-1}-x_{i}\right)+K_{i+1}\left(x_{i+1}-x_{i}\right)-d_{i} v_{i}+u_{i} ;
\end{aligned}
$$

where $x_{0}=x_{10}=0$. Thus, we see this system satisfies our topological assumptions: interconnection of subsystems with local inputs and outputs, only interacting with the neighbors through their state variables.

All masses are taken to be of unit size $M_{i}=1$, and the damping parameter is chosen to be $d_{i}=0.1$ for all masses, but we impose considerable differences on the spring parameters, by choosing $K=$ $[50,40,3,2,1,2,3,4,5,6]$. Intuitively, this suggests a natural way to divide the system into two subsystems, by partitioning through the weakest (5th) spring. We seek to reduce this model while preserving this partition.

Numerical solutions were found using the LMI control toolbox in Matlab. First we attempt to work with the plant directly, without coprime factorization, and seek structured generalized gramians for it, satisfying (2). We find, however, that these LMIs are infeasible; in fact, only for a much more highly damped system $(d=0.7)$ will (2) become feasible. In our lightly damped case, this leads us to consider the coprime factor methods: we tried both the expansive and contractive factorization approaches.

For the expansive case, using our heuristic from Li \& Paganini (2002) to solve the BMIs (4-5), leads to the generalized Hankel singular values

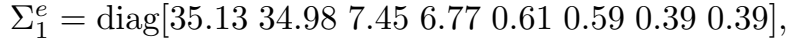

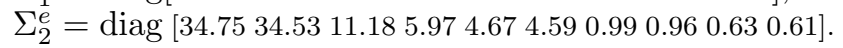

For the contractive method, we get

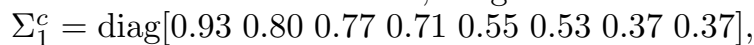

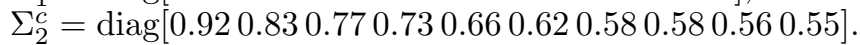

Reducing 2 states from the first subsystem, leads to the following error bounds for each method: $\left\|\left[\begin{array}{c}N^{e} \\ M^{e}\end{array}\right]-\left[\begin{array}{c}N_{r}^{e} \\ M_{r}^{e}\end{array}\right]\right\|_{\infty}=0.7374,\left\|\left[\begin{array}{l}N^{c} \\ M^{c}\end{array}\right]-\left[\begin{array}{c}N_{r}^{c} \\ M_{r}^{c}\end{array}\right]\right\|_{\infty}=0.7372$.

For comparison purposes, we considered doing standard normalized coprime factorization model reduction, reducing 2 states without attention to preserving the topological association of the states. The resulting error in the coprime factors only decreases slightly, to $\left\|\left[\begin{array}{l}N \\ M\end{array}\right]-\left[\begin{array}{l}N_{r} \\ M_{r}\end{array}\right]\right\|_{\infty}=0.7371$. We see that our methods yield comparable errors in the coprime factor sense, but in contrast with the standard coprime factor model reduction method, we are able to preserve the topological structure in terms of the partition of the system states.

As a final point, since as remarked, contractive factorizations do not satisfy a robustness theorem, it is useful to compare the level of " $\gamma$-expansiveness" (as in (11)) 
achieved by our contractive factorization. The result, obtained through a frequency sweep, is $\gamma=0.49$, which gives robust stability for controllers that satisfy a condition approximately twice as tight as that in Lemma 3.

\section{Conclusions}

We have proposed a series of methods for model reduction aimed at preserving underlying topological structure of the plant states, all of which are based on imposing block-diagonal structure to generalized gramians. We have shown how adopting a coprime factor approach greatly extends the range of applicability of this method; to ensure the coprime factorization itself is structurepreserving we use LMI relaxations of the LQ Riccati equation that gives normalized RCFs. Different heuristics are employed to attempt to obtain approximate normalization in this context. We remark that frequencyweighted extensions are also available for this method; for details see Li \& Paganini (2003).

\section{Acknowledgements}

The authors would like to thank the reviewers for their helpful comments.

\section{References}

Anderson, B.D.O., \& Liu, Y. (1989). Controller reduction: concepts and approaches. IEEE Trans. on Autom. Control 34,802-812.

Beck, C.L., Doyle, J.C., \& Glover, K. (1996). Model reduction of multi-dimensional and uncertain systems. IEEE Trans. on Autom. Control 41,1466-1477.

Beck, C.L., \& Bendottii, P. (1997). Model reduction methods for unstable uncertain systems. In Proc. 1997 CDC, pp. 3298-3303.

Dullerud, G.E., \& Paganini, F. (2000). A course in robust control theory : a convex approach. Springer, New York.

El-Zobaidi, H., \& Jaimoukha, I. (1998). Robust control and model and controller reduction of linear parameter varying system. In Proc. 1998 CDC, pp. 3015-3020.

Enns, D.F. (1984). Model reduction with balanced realizations: an error bound and frequency weighted generalization. In Proc. 1984 CDC, pp. 802-812.

Georgiou, T., \& Smith, M. (1990). Optimal robustness in the gap metric. IEEE Trans. on Autom. Control 35,673-686.

Glover, K. (1984). All optimal Hankel-norm approximations of linear multivariable systems and their $L_{\infty}$ error bounds. Int. J. of Control 39,1115-1193.

Li, L., \& Paganini, F. (2002). LMI approach to structured model reduction via coprime factorizations. In Proc. 2002 $A C C$, pp. 1174-1179.

Li, L., \& Paganini, F. (2003). Structured frequency weighted model reduction. In Proc. 2003 CDC, pp. 2841-2846.

Liu, Y., \& Anderson, B.D.O. (1986). Controller reduction via stable factorization and balancing. Int. J. of Control 44,507-531.
McFarlane, D.C., \& Glover, K. (1990). Robust controller design using normalized coprime factor plant descriptions. Springer-Verlag, Berlin; New York.

Meyer, D.G. (1990). Fractional balanced reduction: model reduction via fractional representation. IEEE Trans. Automat. Contr., 35,1341-1345.

Moore, B.C. (1981). Principal component analysis in linear systems: controllability, observablity, and model reduction. IEEE Trans. on Autom. Control 26,17-32.

Tsai,Y.K., Narasimhamurthi,N., \& Wu, F.F. (1982). Structure-preserving model reduction with applications to power system dynamic equivalents. IEEE Trans. on Circuits and Systems, CAS-29,525-535.

Vinnicombe, G. (1993). Frequency domain uncertainty and the graph topology. IEEE Trans. on Autom. Control 38,1371-1383

Wood, G.D., Goddard, P.J., \& Glover, K. (1996). Approximation of linear parameter-varying systems. In Proc. 1996 $C D C$, pp. 406-411.

Zhou, K., D'Souza, C., \& Cloutier, J.R. (1995). Structurally balanced controller order reduction with guaranteed closed loop performance. Systems and Control Letters, 24, 235-242.

Zhou, K., Doyle, J.C., \& Glover, K. (1996). Robust and optimal control. Prentice Hall, Upper Saddle River, N.J.. 